\title{
Evaluation of positioning and density profiling accuracy of muon radiography by utilizing a 15-ton steel block
}

\author{
H. K. M. Tanaka \\ Earthquake Research Institute, the University of Tokyo, Tokyo, Japan
}

Correspondence to: H. K. M. Tanaka (ht@ @riken.jp)

Received: 29 June 2012 - Published in Geosci. Instrum. Method. Data Syst. Discuss.: 21 August 2012

Revised: 8 January 2013 - Accepted: 16 January 2013 - Published: 18 February 2013

\begin{abstract}
A model experiment was performed in order to evaluate the spatial resolution and the accuracy of determining the density by utilizing a steel block. The steel block $\left(7.8 \mathrm{~g} \mathrm{~cm}^{-3}\right)$ is $5 \mathrm{~m}$ thick along the muon trajectories and weighs about $15 \mathrm{t}$. By comparing the experimental result with simulations, the density and the width of the steel block were measured as $7.3 \pm 1.0 \mathrm{~g} \mathrm{~cm}^{-3}$ and $37 \pm 15 \mathrm{~cm}$, respectively.
\end{abstract}

\section{Introduction}

Muon radiography was first proposed to determine a thickness of a rock overburden of a horizontal tunnel in the Snowy Mountains in Australia (George, 1955). He measured the flux inside and outside the tunnel of muons from cosmic ray interactions in the atmosphere and compared them to confirm that a reduction in the muon flux reflects the thickness of the overburden. In his experiment, he compared the muon data with the core sampling data, and concluded that they were consistent within error bars of $\sim 10 \%$.

When applied to a volcano, the method measures the absorption of muons in the volcano projected to the plane of the detector along all muon paths. The amount of energy lost by muons while passing through matter is dependent on the density of that matter. The resultant angular distribution of the muons can then be used to calculate the density profile of the target volume. The change in the muon angle after they pass through a substantial mass is very small $(12 \mathrm{mrad}$ for $\mathrm{SiO}_{2}$ with a thickness of $1.5 \mathrm{~km}$ ). Muon radiography consequently constitutes a unique way to obtain direct information on the density distribution of geological objects with accuracy in terms of spatial resolution that is superior to that possible using conventional geophysical techniques.
The muon is the most abundant of the charged elementary particles at sea level and is capable of traveling large distances through matter. They arrive at angles ranging from the vertical to the horizontal with an integral intensity of $70 \mathrm{~m}^{-2} \mathrm{~s}^{-1} \mathrm{sr}^{-1}$ at sea level. Thus, in order to perform cosmic-ray muon radiography, one makes use either of muons coming from above the structure to be investigated by a detector underneath it, or by quasi-horizontal muons with the detector placed sideways. Since George's first muon radiography, researchers have been attempting to image pyramids (Alvarez et al., 1970), ore bodies (Malmqvist et al., 1979), volcanoes (Tanaka et al., 2007, 2008; Lesparre et al., 2012), seismic faults (Tanaka et al., 2011), caves (Caffau et al., 1997), and archaeological sites (Basset et al., 2006; Menichelli et al., 2007) radiographically using cosmic-ray muons. However, accuracy in positioning and determining the density has not been quantitatively estimated. In this work, these important parameters have been evaluated by using a large steel block whose density and geometry are both well known.

\section{Muon detector}

Conventionally, plastic scintillator strips have been used to assemble two segmented scintillation detector planes to track muon trails (Tanaka et al., 2009; Ambrosi et al., 2011), because a plastic scintillator requires minimal maintenance, has low power consumption, and is relatively insensitive to ambient conditions. Each scintillator strip is coupled with a photomultiplier tube (PMT). A PMT provides high photon sensitivity and fast response to scintillation light, and thus the signal of the PMT can be used as the trigger for timing. In a multi-anode PMT (MAPMT), PMTs are combined so that 
(a)

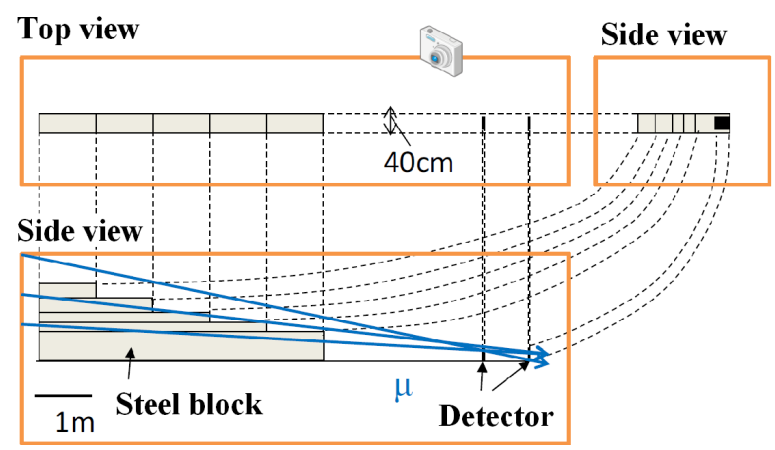

(b)

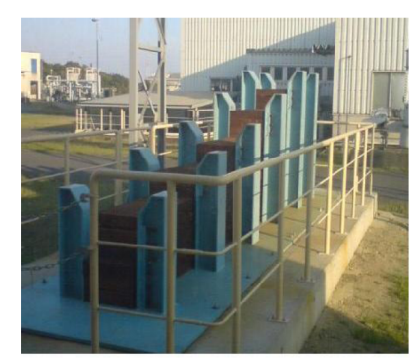

Fig. 1. Schematic view of the experimental setup (a) and a photograph of a 15-t steel block (b).

signals can be treated collectively, and the single scintillator strips do not have to be read out as separate units. Although it is difficult to continue observations when one MAPMT breaks because the detector consists of an integrated combination of scintillators and MAPMTs, its construction can be more robust and better suited for an outdoor environment.

In this work, a segmented muon detection system (Tanaka et al., 2009) was utilized. The system consists of plastic scintillator strips, PMTs, and readout electronics. The size of scintillator strip (Bicron BC-408) is $30 \mathrm{~cm}$ $(L) \times 2.5 \mathrm{~cm}(W) \times 1.5 \mathrm{~cm}(H)$. The plane contains 24 plastic scintillator strips arranged in two arrays along the $x$ and $y$ coordinates, to determine the hit point in the plane by the coincidence of the signals from $x-y$ scintillator strips; the coincidences are registered in a matrix of $12 \times 12$ elements. The total area of the plane is $0.09 \mathrm{~m}^{2}$, and the unit weighs $\sim 10 \mathrm{~kg}$. A slot ( $2 \mathrm{~mm}$ in depth and $2 \mathrm{~mm}$ in width) in the plastic scintillator contains a wavelength-shifting (WLS) fiber with a diameter of $1 \mathrm{~mm}$ (Bicron BCF-91A). The fiber converts purple scintillation light (wavelength $\sim 420 \mathrm{~nm}$ ) into green light (wavelength $\sim 500 \mathrm{~nm}$ ), and conveys the optical signal to an MAPMT. The WLS fiber transmits light with minimal attenuation, because there is a clad layer outside it. The end of the fiber is held by a black colored holder; black silicon rubber is affixed between the fiber and the fiber holder to prevent light leakage. The fiber holder and the MAPMT (HAMAMATSU H8500) are fastened together with light shielding tape. The H8500 MAPMT has 64 anode pixels. While the quantum efficiency of the PMT is $24 \%$ for standard scintillation light, the quantum efficiency is $14 \%$ for the light emitted from the WLS fiber. The quantum efficiency of light emitted from a WLS fiber is therefore $57 \%$ compared with that of the usual scintillation light.

When supplied by $870 \mathrm{~V}$ electric voltage, the typical peak of the pulse height output from the MAPMT was $80 \mathrm{mV}$, and the pulse width was $2 \mathrm{~ns}$. We measured the total electric power consumption by using a power meter (HIOKI 3334 AC/DC POWER HiTESTER) and found that it was $9 \mathrm{~W}$ including the power consumption by the electronics.

\section{Model experiment}

The purpose of the experiment was to estimate the accuracy in positioning and determining the density by using a steel block whose density and geometry are both well known. The procedure includes (1) an estimate of the accuracy in determining the density by assuming the geometry of the steel block is known, and (2) an estimate of the accuracy in positioning by assuming the density of the steel block is known. With this goal, we measured the muon flux using the MAPMT detector and compared the experimental result with a Geant 4 simulation assuming the DEIS flux (the muon flux measured with high statistics at sea-level with the DEIS spectrometer) (Allkofer et al., 1981). Matsuno model (Matsuno et al., 1984) fitted to the DEIS flux was used in this work. The experimental setup is shown in Fig. 1a. The steel block is $5 \mathrm{~m}$ thick along the muon trajectories and weighs about $15 \mathrm{t}$. Its density is $7.8 \mathrm{~g} \mathrm{~cm}^{-3}$. Steel is not pure iron but is an alloy with a variable quantity of carbon ( 0.2 to $2.1 \%$ in weight). Such an alloy can be treated as a chemical mixture at atomic scale. On the other hand, the CSDA (continuous slowing down approximation) range of an $8-\mathrm{GeV}$ muon is $45 \mathrm{hg} \mathrm{cm}^{-2}$ for iron and $39 \mathrm{hg} \mathrm{cm}^{-2}$ for carbon. Therefore, the muon range in pure iron would be slightly longer $(0.26 \%$ at the maximum) than that in steel, but the difference is negligible in this work. The steel block was supported by the metal plate with a thickness of $30 \mathrm{~mm}$ for the purpose of force dispersion. This metal plate was further supported by a concrete block with a thickness of $300 \mathrm{~mm}$ in order to prevent land subsiding. The distance between the center of gravities of the steel block and the detection system is $\sim 5 \mathrm{~m}$. The distance between two segmented scintillation detector planes was $75 \mathrm{~cm}$, and the root mean square (RMS) angular resolution of the system was $\pm 14 \mathrm{mrad}$ at an angular interval of $33 \mathrm{mrad}$. The RMS angular resolution $\left(\Delta \theta_{\text {res }}\right)$ is the minimum resolvable angle of the detector, and the angular interval $\left(\Delta \theta_{\text {int }}\right)$ is the ratio of the scintillator strips $(d)$ and the distance between two detector planes $(L), d / L . \Delta \theta_{\text {res }}$ and $\Delta \theta_{\text {int }}$ have the following relationship: 


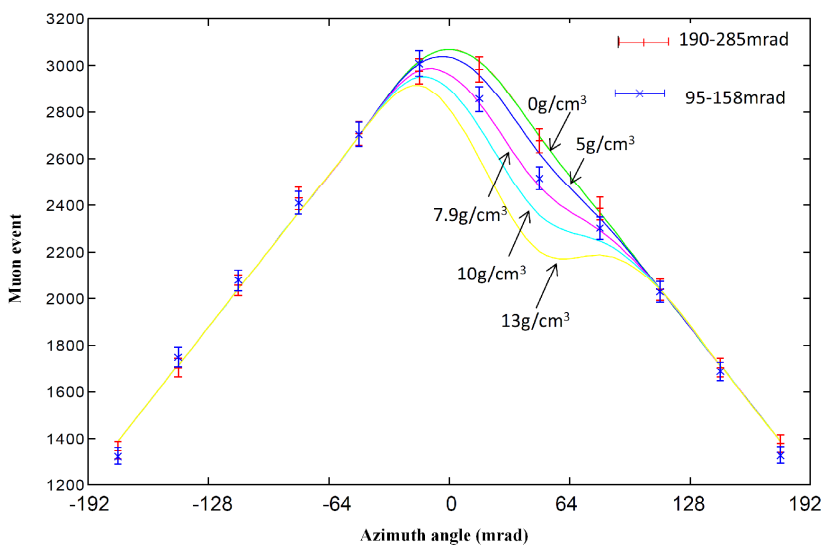

Fig. 2. Muon events as a function of azimuth angles counted within different elevation angle regions $(95<\theta<158 \mathrm{mrad}$ and $190<\theta<285 \mathrm{mrad})$. Monte Carlo simulation results for different uniform densities $\left(0,5,7.9,10\right.$ and $\left.13 \mathrm{~g} \mathrm{~cm}^{-3}\right)$ are overlaid on the data.

$\left(\Delta \theta_{\text {int }}-\Delta \theta_{\text {res }}\right)^{2} / \Delta \theta_{\text {int }}^{2}=0.32$. The muon data were compiled and analyzed by a network-based FPGA (field programmable gate array) muon readout electronics (Uchida et al., 2010). The FPGA works on a 50-MHz system clock. The event filter (which selects events that can be used to construct the muon paths and generates path information), the histogram generator, and the network processor are implemented on the single FPGA. The PMT signals analyzed in the FPGA chip are recorded in a number of bins representing the azimuth and elevation arriving angles of cosmic ray muons. The data were read by the network processor when a remote PC accessed the electronics. The observation period was one month.

In order to compare the experimental result with simulations, we performed Monte Carlo simulations by taking the following steps.

1. 3-D geometrical information of the steel block was used to obtain the path length in the direction of $\theta$ and $\varphi$, as seen from the muon detector by constructing a virtual detection system on a computer.

2. We generated random numbers, and using these numbers we generated muons adapted to the energy distribution obtained by Eq. (1).

3. The generated muons were injected into a steel block with a thickness of $X(\mathrm{~m})$ in order to calculate the stopping length of the muons in the target by using Geant 4 (Agostinelli et al., 2003), and a table $N(\theta, X)$ was constructed to represent the data.

4. By using the result of (1) and (3), we calculated the number of muons during a 30-day measurement period counted in direction of $\theta$ and $\varphi$.

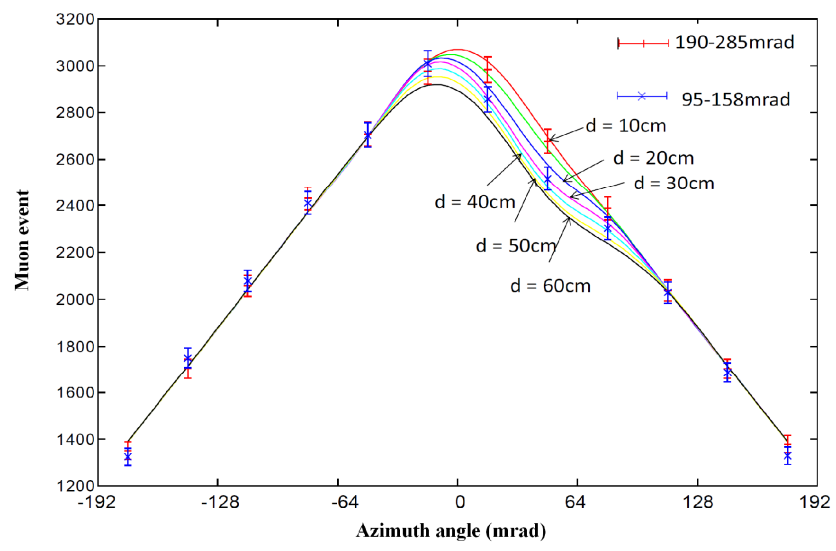

Fig. 3. Muon events as a function of azimuth angles counted within different elevation angle regions $(95<\theta<158 \mathrm{mrad}$ and $190<\theta<285 \mathrm{mrad})$. Monte Carlo simulation results for various widths $(10,20,30,40,50$ and $60 \mathrm{~cm})$ of the steel block are overlaid on the data.

\section{Results and discussions}

The result of a 30-day measurement period is shown in Fig. 2. The numbers of muon events counted within the different elevation angular regions of $95 \mathrm{mrad}<\theta<158 \mathrm{mrad}$ and $190 \mathrm{mrad}<\theta<285 \mathrm{mrad}$ are plotted with bars and crosses respectively. The whole area of the detection system is sensitive to muons that arrive near horizontally, but the system does not catch all the muons that enter at large angles to the horizontal. As shown in Fig. 2, the system has a geometrical acceptance, which can be used to correct the horizontal distributions of muons. In this figure, the geometrical acceptance was corrected for the elevation angles. The muons with angles 190-285 mrad have not passed through the block, while those with angles 95-158 have. The plots for $190 \mathrm{mrad}<\theta<285 \mathrm{mrad}$ show the data without a target object, and the results confirmed the integral muon intensity as can be calculated by Eq. (1). For $95 \mathrm{mrad}<\theta<158 \mathrm{mrad}$, significant decreases in events can be seen between $\varphi=0$ and $66 \mathrm{mrad}$, primarily due to increases in the average density along the muon paths coming from an existence of the steel block.

This result was compared with the Geant 4 Monte Carlo simulations (solid lines in Fig. 2), which give the integrated flux of muons at various azimuth angles penetrating through a given density length (density $\times$ path length) by referring to the geometry of the steel block. The simulations were performed by assuming the uniform average density values that range from 0 to $13 \mathrm{~g} \mathrm{~cm}^{-3}$. From the comparison between the experimental and simulation result, the average density of the steel block was determined as $7.3 \pm 1.0 \mathrm{~g} \mathrm{~cm}^{-3}$ at a $1 \sigma$ confidence level. Figure 3 shows the integrated flux of muons penetrating through a given geometry of the steel block by referring to the average density of the steel block, which was determined in the previous procedure. By comparing the data 
with the simulation results for different widths of the steel block, which ranges from 10 to $60 \mathrm{~cm}$, the width was determined as $37 \pm 15 \mathrm{~cm}$ at a $1 \sigma$ confidence level.

The standard deviation was computed by assuming Poissonian statistics (Lesparre et al., 2010). Therefore, the standard deviation can be significantly reduced by making measurements over a longer period. Also, the accuracy will be improved when the target is thicker and adequate statistics is available (Fig. 4). Since the average energy of quasihorizontal muons ranges from 10 to $30 \mathrm{GeV}$, the accuracy will be improved when the target size is slightly bigger than that used in this work (the range of $30-\mathrm{GeV}$ muons is $150 \mathrm{hg} \mathrm{cm}^{-2}$ in iron). This is consistent with an uncertainty of $0.2 \mathrm{~g} \mathrm{~cm}^{-3}$ that is announced for a measurement period of 45 days (Nagamine et al., 2005).

In this experiment, the density of the steel block was determined as $7.3 \pm 1.0 \mathrm{~g} \mathrm{~cm}^{-3}$; therefore, the accuracy in determining the density is $13 \%$. The typical energy of muons at sea level is $3-4 \mathrm{GeV}$, sufficient to penetrate meters of steel block. Most of the muons go through the steel block, and thus the absorption rate is not very sensitive to the target density. However, a similar experiment with $1 \mathrm{~km}$ rock and adequate statistics gives a more accurate result. Figure 4 shows a simulation result of the sensitivity of the muon transmission rate to the target density for different path lengths. The simulation compares the transmission rate between a $1-\mathrm{km}$ rock $\left(\rho=2 \mathrm{~g} \mathrm{~cm}^{-3}\right)$ and a 5 -m steel block $\left(\rho=7.9 \mathrm{~g} \mathrm{~cm}^{-3}\right)$. Since the low energy muon flux is screened by the target volume, the density can be more precisely determined for a thicker target. The statistical error in the measured number of muons in the present experiment was $\pm 2 \%$. For this variation, we can calculate that the accuracy in determining the density of $1 \mathrm{~km}$ of rock is $\pm 1 \%$, whereas that for a $5-\mathrm{m}$ steel block is $\pm 13 \%$.

Although the low density found for the steel block (7.3 instead of $7.8 \mathrm{~g} \mathrm{~cm}^{-3}$ ) is within the error, it is possibly attributed to background noise due to fake tracks produced by the accidental coincidences of vertical electromagnetic shower particles. Such events are likely to occur in a twoplane system as used in the experiments in this work. This effect has to be considered when the target size is larger.

The RMS angular resolution of the present system is $\left(\Delta \theta_{\text {res }}, \Delta \varphi_{\text {res }}= \pm 14 \mathrm{mrad}, \pm 14 \mathrm{mrad}\right)$ at an angular interval of $33 \mathrm{mrad}$. The minimum resolvable distance (MRD) (spatial resolution $\Delta X_{\text {res }}, \Delta Y_{\text {res }}$ ) at the target volume (in this case, an Fe block) is defined by the minimum resolvable angle of the detector (in this case, the RMS angular resolution) ( $\left.\Delta \theta_{\text {res }} \Delta \varphi_{\text {res }}\right)$ and the distance between the target and the detector $(R):\left(\Delta X_{\text {res }}, \Delta Y_{\text {res }}\right)=R \times\left(\Delta \theta_{\text {res }}, \Delta \varphi_{\text {res }}\right)$. The spatial interval $\left(\Delta X_{\text {int }}, \Delta Y_{\text {int }}\right)$ at the target volume is defined by the angular interval of the detector $\left(\Delta \theta_{\text {int }} \Delta \varphi_{\text {int }}\right)$ and the distance between the target and the detector $(R):\left(\Delta X_{\mathrm{int}}\right.$, $\left.\Delta Y_{\text {int }}\right)=R \times\left(\Delta \theta_{\text {int }}, \Delta \varphi_{\text {int }}\right)$. Therefore we expect that the theoretical MRD will be $\pm 7.0 \mathrm{~cm}$ with a spatial interval of $16.5 \mathrm{~cm}$ in the present case. In this work, the actual minimum

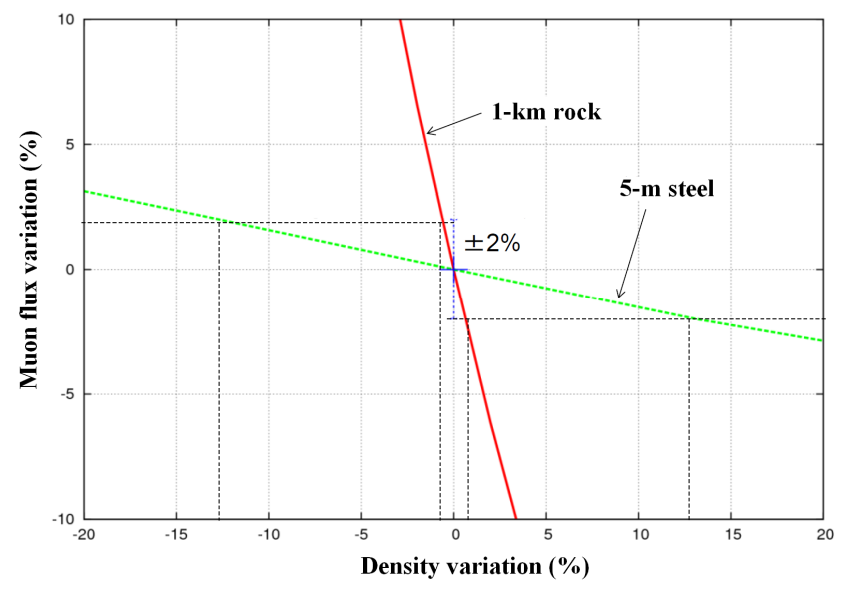

Fig. 4. Simulation results of the sensitivity of the muon transmission rate to the target density for different path lengths.

resolvable distance $(\Delta X=15 \mathrm{~cm})$ was measured between the theoretical MRD and the spatial interval:

$\Delta X_{\text {res }}<\Delta X<\Delta X_{\text {int }}$

Equation (1) confirms that the theoretical MRD (RMS angular resolution) of the segmented muon detection system approximately equals the actual MRD (or spatial resolution) at the target. Tanaka et al. $(2007,2008,2009,2011)$ and Lesparre et al. (2012) used one half of the spatial interval as the minimum resolvable distance. They both used a segmented muon detection system. Their approaches were validated by this work.

\section{Conclusions}

The accuracy of positioning and determining densities in muon radiography was first quantitatively evaluated by utilizing a steel block. In the present experiment, we measured an accuracy of $13 \%$ in determining the density of a steel block with a length of $5 \mathrm{~m}$, and based on this value we confirmed that the accuracy will be improved to $1 \%$ when the target is a rock with a thickness of $1 \mathrm{~km}$ and adequate statistics is available. Also, we confirmed that the positioning resolution depends on the angular resolution of the detection system.

Acknowledgements. The experiment was supported by a collaborative research between Nippon Steel Corporation and University of Tokyo. Analysis and simulation works greatly benefited from the skill and expertise of Hideaki Taira. The manuscript benefited from useful comments provided by T. Hurst, D. Gibert, and anonymous reviewers.

Edited by: T. Hurst 


\section{References}

Agostinelli, S., Allison, J., Amako, K., Apostolakis, J., Araujo, H., Arce, P., Asai, M., Axen, D., Banerjeel, S., Barrand, G., Behner, F., Bellagamba, L., Boudreau, J., Broglia, L., Brunengo, A., Burkhardt, H., Chauvie, S., Chuma, J., Chytrace, R., Cooperman, G., Cosmo, G., Degtyarenko, P., Dell'Acqua, A., Depaola, G., Dietrich, D., Enami, R., Feliciello, A., Ferguson, C., Fesefeldt, H., Folger, G., Foppiano, F., Forti, A., Garelli, S., Giani, S., Giannitrapani, R., Gibin, D., Gómez Cadenas, J. J., González, I., Gracia Abril, G., Greeniaus, G., Greiner, W., Grichine, V., Grossheim, A., Guatelli, S., Gumplinger, P., Hamatsu, R., Hashimoto, K., Hasui, H., Heikkinen, A., Howard, A., Ivanchenko, V., Johnson, A., Jones, F. W., Kallenbach, J., Kanaya, N., Kawabata, M., Kawabata, Y., Kawaguti, M., Kelner, S., Kent, P., Kimura, A., Kodama, T., Kokoulin, R., Kossov, M., Kurashige, H., Lamanna, E., Lampén, T., Lara, V., Lefebure, V., Lei, F., Liendl, M., Lockmanj, W., Longo, F., Magni, S., Maire, M., Medernach, E., Minamimoto, K., Mora de Freitas, P., Morita, Y., Murakami, K., Nagamatu, M., Nartallo, R., Nieminen, P., Nishimura, T., Ohtsubo, K., Okamura, M., O’Neale, S., Oohata, Y., Paech, K., Perl, J., Pfeiffer, A., Pia, M. G., Ranjard, F., Rybin, A., Sadilov, S., Di Salvo, E., Santin, G., Sasaki, T., Savvas, N., Sawada, Y., Scherer, S., Sei, S., Sirotenko, V., Smith, D., Starkov, N., Stoecker, H., Sulkimo, J., Takahata, M., Tanaka, S., Tcherniaev, E., Safai Tehrani, E., Tropeano, M., Truscott, P., Uno, H., Urban, L., Urban, P., Verderi, M., Walkden, A., Wander, W., Weber, H., Wellisch, J. P., Wenaus, T., Williams, D. C., Wright, D., Yamada, T., Yoshida, H., and Zschiesche, D.: Geant4-a simulation toolkit, Nucl. Instrum. Meth. A, 506, 250-303, 2003.

Allkofer, O. C., Carstensen, K., Dau, W. D., Jokisch, H., Klemke, G., Oren, Y., and Uhr, R. C.: Muon Spectra from DEIS up to $7 \mathrm{TeV}$, Proc. of 17th Cosmic Ray Conf., Paris, France, 10, p. 321, 1981.

Alvarez, L. W., Anderson, J. A., Bedwei, F. E., Burkhard, J., Fakhry, A., Girgis, A., Goneid, A., Hassan, F., Iverson, D., Lynch, G., Miligy, Z., Mousaa, A. H., Sharkawi, M., and Yazolinio, L.: Search for hidden chambers in the pyramids, Science, 167, 832839, 1970.

Ambrosi, G., Ambrosino, F., Battiston, R., Bross, A., Callier, S., Cassese, F., Castellini, G., Ciaranfi, R., Cozzolino, F., D’Alessandro, R., de La Taille, C., Iacobucci, G., Marotta, A., Masone, V., Martini, M., Nishiyama, R., Noli, P., Orazi, M., Parascandolo, L., Parascandolo, P., Passeggio, G., Peluso, R., Pla-Dalmau, A., Raux, L., Rocco, R., Rubinov, P., Saracino, G., Scarpato, G., Sekhniaidze, G., Strolin, P., Tanaka, H. K. M., Tanaka, M., Trattino, P., Uchida, T., and Yokoyamao, I.: The MU-RAY project: Volcano radiography with cosmic-ray muons, Nucl. Instrum. Meth. A, 628, 120-123, 2011.

Basset, M., Ansoldi, S., Bari, M., Battiston, R., and Blasko, S.: MGR: An innovative, low-cost and compact cosmic-ray detector, Nucl. Instrum. Meth. Phys. Res. A, 567, 298-301, 2006.

Caffau, E., Coren, F., and Giannini, G.: Underground cosmic-ray measurement for morphological reconstruction of the "Grotta Gigante" natural cave, Nucl. Instrum. Meth. Phys. Res. A, 385, 480-488, 1997.
George, E. P.: Cosmic rays measure overburden of tunnel, Commonwealth Engineer, Tait/Melbourne, 455-457, 1955.

Lesparre, N., Gibert, D., Marteau, J., D’eclais, Y., Carbone, D., and Galichet, E.: Geophysical muon imaging: feasibility and limits, Geophys. J. Int., 183, 1348-1361, 2010.

Lesparre, N., Gibert, D., Marteau, J., Komorowski, J., Nicolin, F., and Coutant, O.: Density muon radiography of La Soufrière of Guadeloupe volcano: comparison with geological, electrical resistivity and gravity data, Geophys. J. Int., 190, 1008-1019, doi:10.1111/j.1365-246X.2012.05546.x, 2012.

Malmqvist, L., Jonsson, G., Kristiansson, K., and Jacobsson, L.: Theoretical studies of in-situ rock density determination using cosmic-ray muon intensity measurements with application in mining geophysics, Geophysics, 44, 1549-1569, 1979.

Matsuno, S., Kajino, F., Kawashima, Y., Kitamura, T., Mitsui, K., Muraki, Y., Ohashi, Y., Okada, A., Suda, T., Minorikawa, Y., Kobayakawa, K., Kamiya, Y., Nakamura, I., and Takahashi, T.: Cosmic-ray muon spectrum up to $20 \mathrm{TeV}$ at $89^{\circ}$ zenith angle, Phys. Rev. D, 29, 1-23, 1984.

Menichelli, M., Ansoldi, S., Bari, M., Basset, M., and Battiston, R.: A scintillating fibres tracker detector for archaeological applications, Nucl. Instrum. Meth. Phys. Res. A, 572, 262-265, 2007.

Nagamine, K., Tanaka, H. K. M., Nakamura, S. N., Ishida, K., Hashimoto, M., Shinotake, A., Naito, M., and Hatanaka, A.: Probing the inner structure of blast furnaces by cosmic-ray muon radiography, P. Jpn. Acad. B-Phys., 81, 257-260, 2005.

Tanaka, H. K. M., Nakano, T., Takahashi, S., Yoshida, J., Takeo, M., Oikawa, J., Ohminato, T., Aoki, Y., Koyama, E., Tsuji, H., and Niwa, K.: High resolution imaging in the inhomogeneous crust with cosmic ray muon radiography: The density structure below the volcanic crater floor of Mt. Asama, Japan, Earth Planet. Sc. Lett., 263, 104-113, 2007.

Tanaka, H. K. M., Nakano, T., Takahashi, S., Yoshida, J., Takeo, M., Oikawa, J., Ohminato, T., Aoki, Y., Koyama, E., Tsuji, H., Ohshima, H., Maekawa, T., Watanabe, H., and Niwa, K.: Radiographic imaging below a volcanic crater floor with cosmic-ray muons, Am. J. Sci., 308, 843-850, 2008.

Tanaka, H. K. M., Uchida, T., Tanaka, M., Shinohara, H., and Taira, H.: Cosmic-ray muon imaging of magma in a conduit: Degassing process of Satsuma-Iwojima Volcano, Japan, Geophys. Res. Lett., 36, L01304, doi:10.1029/2008GL036451, 2009.

Tanaka, H. K. M., Miyajima, H., Kusagaya, T., Taketa, A., Uchida, T., and Tanaka, M.: Cosmic muon imaging of hidden seismic fault zones: Rainwater permeation into the mechanical fractured zones in Itoigawa-Shizuoka Tectonic Line, Japan, Earth Planet. Sc. Lett., 306, 156-162, 2011.

Uchida, T., Tanaka, H. K. M., and Tanaka, M.: Development of a muon radiographic imaging electronic board system for a stable solar power operation, Earth Planets Space, 62, 167-172, 2010. 